\title{
Dimensional Synthesis of Wristed Binary Hands
}

\author{
Neda Hassanzadeh \\ Department of Mechanical Engineering, \\ Idaho State University \\ 921 S. 8th. Ave, Pocatello, ID 83209, USA \\ e-mail:hassnedalisu.edu \\ Alba Perez-Gracia \\ Associate Professor \\ Department of Mechanical Engineering, \\ Idaho State University \\ 921 S. 8th. Ave, Pocatello, ID 83209, USA \\ e-mail:perealbaeisu.edu
}

\begin{abstract}
The kinematic synthesis applied to tree topologies is a tool for the design of multi-fingered robotic hands, for a simultaneous task of all fingertips. Even though traditionally wrists and hands have been designed separately, the wrist usually being part of the robot manipulator arm, it makes sense to consider the wrist as a part of the hand, as many grasping and manipulation actions are a coordinated action of wrist and fingers. The manipulation capabilities of robotic hands may also be enhanced by considering more than one splitting stage, as opposed to the single-palm traditional hand.

In this work we present the dimensional synthesis for a family of multi-fingered hands, the binary hands, which have a $2 R$ wrist and several splitting stages, each of them spanning two branches consisting $f$ a revolute joint for each edge. For these topologies, it is proved that a three-position task can be defined for each fingertip, regardless of the number of fingers. One example is presented to show the possible design strategies and uses for this family of hands.
\end{abstract}

\author{
Nomenclature \\ S A line or dual vector, defined using Plucker coordinates. \\ s A vector \\ $\hat{S} \quad$ A quaternion or dual quaternion \\ $\hat{w} \quad$ A dual number \\ $[M] \quad$ A matrix
}

\section{Introduction}

The topology of an articulated system defines the number and type of joints of the system, their adjacency, and their incidence on the end-effectors. In dimensional kinematic synthesis, the goal is to determine the location and orientation of the joint axes of a given topology so that it can perform a given task. It has only been recently that kinematic synthesis has been applied to multiple end-effector systems. These systems can be simplified, for synthesis purposes, to chains with a tree topology, with several common joints that branch to a number of serial chains, and possibly with several branching stages, ending with several end-effectors. A typical example of a kinematic chain with a tree topology is a wristed, multi-fingered hand.

Kinematic analysis of tree topologies for applications in modular robots and robotic hands can be found in [1], [2], and [3], and dynamic analysis is found in [4] and [5]. Structural synthesis for multiple fingers with no wrist is also considered in [2], and in [6] for grasping and manipulation requirements.

The dimensional synthesis of the tree topologies presents particular challenges that are different from those that appear in single serial chains or in closed-loop systems. In particular, the dimensional synthesis of multi-fingered hands has been explored in [7], [8] and more extensively in [9]. In this case, the kinematic task is defined as having the same number of positions for each of the multiple endeffectors; this means that we are dealing with a coordinated action of all those end-effectors, denoted as a simultaneous task.

The synthesis of tree topologies, when applied to wristed multi-fingered hands, allows creating robotic hands for in-palm manipulation tasks, for instance, or complete arm-plus-hand designs. The choice of the topology so that it is more suited for the task is the object of the type, or structural, synthesis.

A first attempt at the enumeration of tree topologies for the design of multi-fingered hands can be found in [10], where solvability was studied. In this study it was concluded that there are some families of topologies for which the number of fingers can be increased arbitrarily without constrain- 
ing their ability to perform a task.

In this paper we focus on one family of tree topologies, denoted as binary hands, which is part of the bigger family of fractal hands. For this family, the topologies are enumerated and characterized, solvability is studied and the dimensional synthesis is solved for the $2 \mathrm{R}$ wrested hand with $\mathrm{R}$ joint edges. A closed algebraic solution is derived and the number of solutions is calculated for any number of fingers. Finally, one example is presented that show the potential of this family of multi-fingered hands.

\section{Multi-fingered Robotic Hands}

Multi-fingered robotic hands consist, in the most general case, of a series of common joints spanning several kinematic chains, which can split again in new chains, up to a set of end-effectors, or fingertips [11]. We define a branch of the hand as the serial chain connecting the root node to one of the end-effectors, and a palm as a link that is ternary or above. In this work it is considered that the fingertips are the only elements whose motion or contact with the environment is being defined by the task; this could be generalized by considering also other intermediate links of the hand.

These tree topologies are represented as rooted tree graphs; for this we follow the approach of Tsai [12], the root vertex being fixed with respect to a reference system. In tree topologies, a vertex can be connected to several edges defining several branches. Most of the current robotic hands have one palm and several fingers, and may have a few wrist joints. Figure 1 shows a commercial hand, the Barret ${ }^{\circledR}$ Hand, and a modeling of the human hand considering the wrist, with their tree representations.

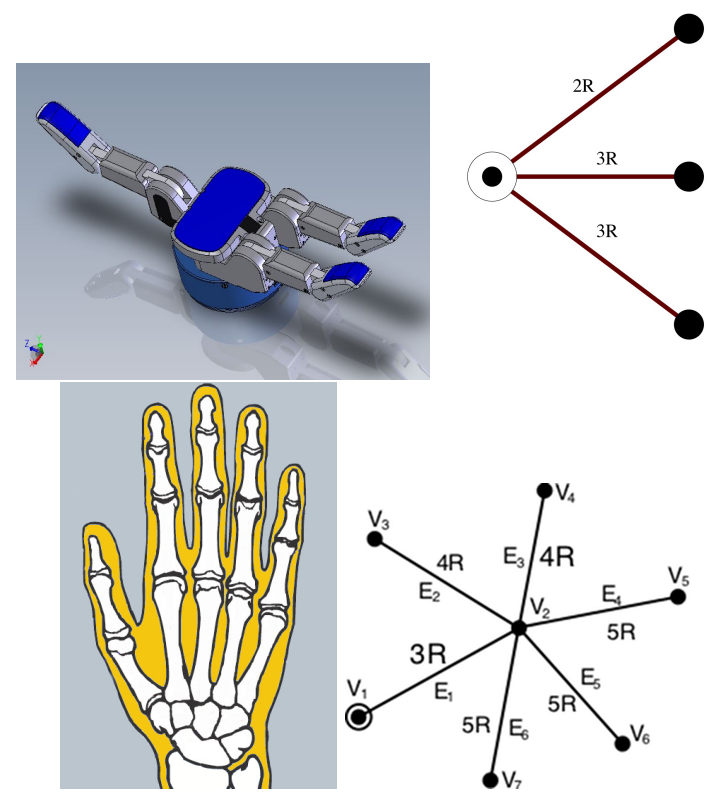

Fig. 1. Barrett ${ }^{\circledR}$ Hand, wristed human hand and their tree representations. Vertices are labeled as $\mathrm{V}$, edges as $\mathrm{E}$, and $\mathrm{R}$ denotes revolute joints.
Open hands, that is, hands not holding an object in the fingers, are kinematic chains with a tree or hybrid topology. For our synthesis formulation, the internal loops in the hand structure are substituted using a reduction process [9], so that the hand has a tree topology with links that are ternary or above.

Tree topologies are denoted as $S C-\left(B_{1}, B_{2}, \ldots, B_{b}\right)$, where $S C$ indicates the common joints and the dash indicates a branching or splitting, with the branches contained in the parenthesis, each branch $B_{i}$ characterized by its type and number of joints. In the case of using just revolute joints, the joint type is dropped and only the number of joints is indicated. For Figure 1, the tree topologies would be denoted as $0-(2,3,3)$ and $3-(4,4,5,5,5)$ respectively.

Our definition of multi-fingered hand considers the wrist as a part of the hand, as many grasping and manipulation actions are a coordinated action of wrist and fingers. In addition, the hand design may present several splitting stages, such as the $2-(2,1-(3,3,3), 2)$ tree topology shown in Figure 2 , with a $2 \mathrm{R}$ wrist and two splitting stages.

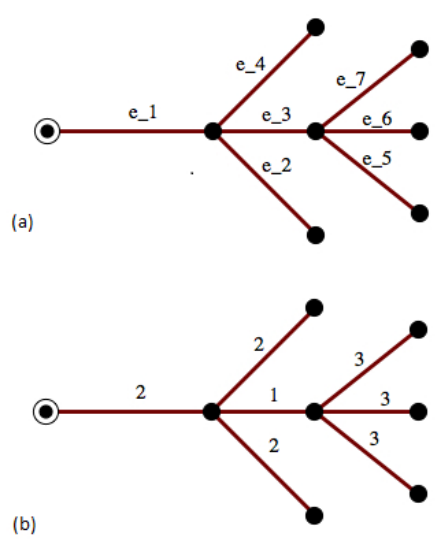

Fig. 2. A tree topology with two splitting stages. (a) edge labeling; (b) number of joints in each edge.

Two arrays can be associated to a hand topology. One of them is the parent-pointer representation, in which the value of the array for each labeled edge corresponds to the previous edge of the graph. The other array is the joint array, which contains the number of joints for each edge. For instance, for the tree graph in Figure 2, the parent-pointer array is $p t=$ $\{0,1,1,1,3,3,3\}$ and the joint array is $j=\{2,2,1,2,3,3,3\}$, according to the edge notation of the Figure.

\section{Dimensional Synthesis for Multi-fingered Hands}

Given a hand topology and a kinematic task consisting of a set of finite positions for each end-effector, dimensional kinematic synthesis seeks to find the position of the joint axes in order for each of the end-effectors to perform the given set of displacements simultaneously; see [9]. In this section, the formulation of the synthesis equations and the calculation of the solvability of the topology are described. 


\subsection{Dimensional Synthesis Equations}

The approach followed to create dimensional synthesis equations consists on equating the forward kinematics of each root-to-fingertip branch in the hand to the set of positions defined for that particular fingertip, which is the endeffector. Given a set of $m$ task positions $\hat{P}_{k}^{i}, k=1 \ldots m$, for each end-effector (denoted by superscript $i$ ), we compute the relative displacements from a selected reference position (let us say, position 1), and equate the relative forward kinematics to those relative positions. For a hand with $b$ fingertips, this yields $b$ sets of equations that are to be solved simultaneously,

$$
\hat{P}_{1 k}^{i}=\prod_{j \in\left\{B_{i}\right\}} e^{\frac{\Delta \hat{\theta}_{j}^{k}}{2} \mathrm{~S}_{j}}, \quad i=1, \ldots, b ; \quad k=2, \ldots, m,
$$

where the number of end-effectors, or branches as root-tofingertip chains, is indicated by $b$, and $\left\{B_{i}\right\}$ is the set of ordered indices of the joints belonging to branch $i$. Notice that some of the joints will be common to several branches. The joint axes at the reference configuration are denoted as $S_{j}$.

This yields a total of $6(m-1) b$ independent equations to be simultaneously solved.

\subsection{Solvability of Tree Topologies for Exact Synthesis}

We define a kinematic chain as solvable [10] if we can find a positive rational number of positions for which the exact dimensional synthesis yields a finite number of solutions. In the case of multiple end-effectors, the system of equations needs to be properly dimensioned to be solved simultaneously, while not overconstraining any of the branches. This translates to checking the relative solvability for all possible subgraphs starting at a root node and ending at -at least- one end effector, including all possible root node changes.

The number of positions for each subgraph is computed as follows: let $\mathbf{D}_{j}^{e}$ be an $e \times 1$ vector containing the joint degrees-of-freedom for each edge of the contracted graph, and $\mathbf{D}_{s}^{e}$ be the $e \times 1$ vector containing the number of structural parameters (four per joint in the general case) for each edge of the contracted graph. Denote as $\mathbf{D}_{e e}^{n}$ the $b \times 1$ vector containing the degrees-of-freedom of the space of each endeffector, and $\mathbf{D}_{c}^{n}$ the $b \times 1$ vector with the number of additionally imposed constraints (if any) for each branch. Define the vectors $\mathbf{B}_{i}$ as a $b_{i} \times 1$ vector of ones corresponding to the branches included in the graph, and $\mathbf{E}_{i}$ as an $e_{i} \times 1$ vector of ones for the edges included in the graph considered. Here the subscript $i$ indexes the set of subgraphs starting at the root node and ending in at least one end-effector, for a given tree topology. These last two vectors can be easily computed from the end-effector path matrix and the incidence matrix of the overall graph, see [9]. The number of positions for exact synthesis of the graph or subgraph $i$ is given by

$$
m_{i}=\frac{\mathbf{D}_{s}^{e} \cdot \mathbf{E}_{i}-\mathbf{D}_{c}^{n} \cdot \mathbf{B}_{i}}{\mathbf{D}_{e e}^{n} \cdot \mathbf{B}_{i}-\mathbf{D}_{j}^{e} \cdot \mathbf{E}_{i}}+1
$$

An overall solution can be found, for arbitrary tasks, only when considering the solvability of all subgraphs that start at the root node and end at end-effectors, including all subgraphs obtained when exchanging the root node with one of the end effectors as described above. In this case, considering $m$ as the number of positions for exact synthesis of the overall graph and $m_{i}$ as the number of positions for exact synthesis for a subgraph $i$, with $i \in S$ the set of all possible different end-effector subgraphs up to isomorphism, the topology is solvable if

1. $m \in \mathbb{Q}^{+}$

2. $m \leq m_{i}, \forall m_{i} \in \mathbb{Q}^{+}, i \in \mathrm{S}$

In the case of a subgraph containing $c$ branches and being solvable for $m_{i}=m$ positions, that subgraph can be solved separately, which eliminates exactly $6 c(m-1)$ equations and the same number of unknowns, so that the rest of the graph can be solved a posteriori.

\section{Binary Hands}

We denote as binary hands those hands that are represented by a symmetric binary tree, that is, a tree in which each vertex spans two edges, up to a given level. This is a particular case of the more general family of fractal hands, in which $k$ branches are spanned at each splitting point. Partial fractal hands have been mentioned in [13]; see Figure 3 for some examples.
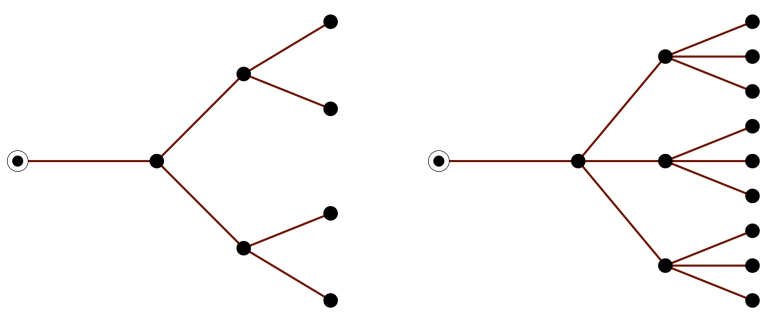

Fig. 3. Graphs for some wristed fractal hands: binary and ternary.

In our case, the symmetry of the binary tree is broken at the wrist; the first edge consists of a serial chain with two revolute joints, while the rest of edges are single revolute joints. The structure of these binary trees allows us to define their topology using the number of branching stages, or splitting stages, of the tree. If a tree has depth $s+1$ (depth of a tree is defined as the number of edges from the root to the end-effector node), then the number of splitting stages is $s$, because there is no splitting stage for the wrist. Figure 4 


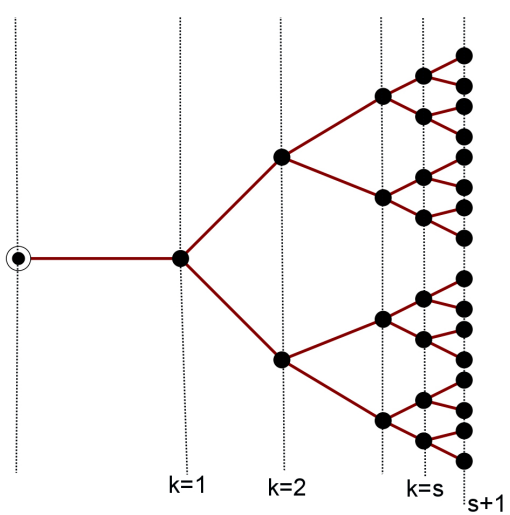

Fig. 4. For a tree with depth $s+1$, the number of splitting stages is $s$, as there is no splitting at the wrist.

shows the relation between the depth and the splitting stages of the binary trees.

For a wristed, binary hand with $s$ splitting stages,

1. The total number of splits is $2^{s}-1$,

2. The total number of edges is $2^{s+1}-1$,

3. The hand has $b=2^{s}$ end-effectors, or fingertips,

4. The parent-pointer representation, when naming the edges as they appear sequentially from the root vertex, is $p t=\left\{0,1,1,2,2,3,3,4,4, \ldots, 2^{s}-1,2^{s}-1\right\}$.

The total number of joints of the hand is hence $2^{s+1}$ counting the two joints at the common wrist edge. Figure 5 shows four examples of binary hands, with one to four splitting stages.
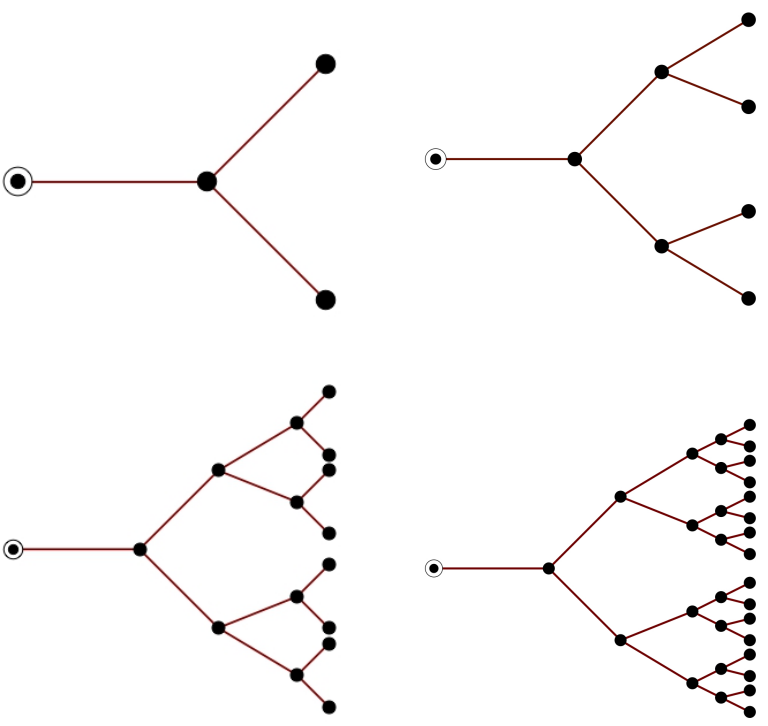

Fig. 5. Graphs for binary trees with one, two, three and four splitting stages.

Wristed binary hands may be used for scalable interaction with the environment. Figure 6 below shows a $1-(1-$
$(1,1), 1-(1,1))$ binary hand with a planar motion at the four fingertips that can be combined to create two small loppers or a larger one, using two end-effectors for each one. In this case, the task defines the workspace of the wrist, so that finger actions can be performed within a given volume and for given orientations.

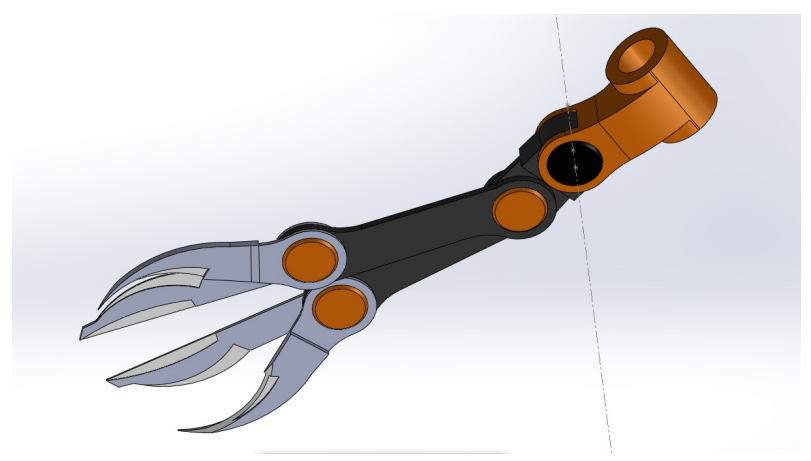

Fig. 6. An example of a two-stage binary hand

\subsection{Partial Binary Hands}

Partial fractal hands, in which the symmetry is broken at a given splitting stage by eliminating, or pruning, some of the branches, may be also considered as suitable topologies for the design of multi-fingered robotic hands.

In the case of binary hands, it is possible to eliminate up to $2^{k}$ new branchings with their subsequent edges at each depth $k$ of the tree, with $k=1$ to $s$. Because of solvability issues presented in subsequent sections, the case studied here is that of eliminating both edges incident in a given vertex, for a certain number of vertices $r \leq 2^{k-1}$. In particular, for a binary hand of depth $s+1$ in which we eliminate both edges incident on a single vertex at a given depth $k$, we are eliminating:

1. A total of $2^{s+1-k}-1$ splits,

2. A total of $2\left(2^{s+1-k}-1\right)$ edges,

3. A total of $2^{s+1-k}$ end-effectors.

This counting can be generalized to the case in which we eliminate $r_{1}$ pairs of branches at a depth $k_{1}, r_{2}$ pairs of branches at a depth $k_{2}$, etcetera, so that the number of endeffectors eliminated will be $r_{1} 2^{s+1-k_{1}}+r_{2} 2^{s+1-k_{2}}+\ldots$ A new end-effector is created for each pair eliminated, so that the total number of end-effectors for a partial binary hand of depth $s+1$ in which $p$ prunings are performed is

$$
b=2^{s}\left(1-\sum_{i=1}^{p} r_{i} 2^{1-k_{i}}\right)+p .
$$

Figure 7 shows the partial binary hand with one pair of branches removed at depth 2 and another pair removed at depth 3 . 


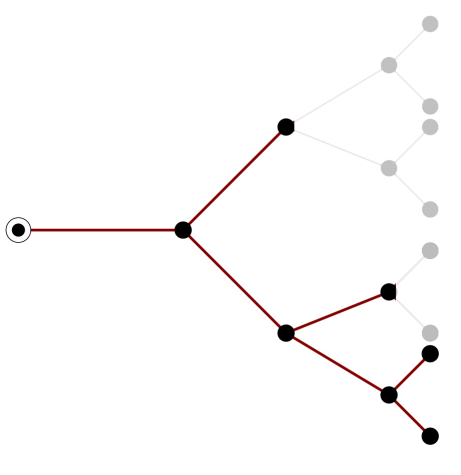

Fig. 7. An binary tree pruned at depth 2 and depth 3 , with a total of 4 end-effectors.

\subsection{Other Fractal Hands}

The characterization of symmetric, fractal tree topologies can be generalized to any number $k$ of new branches per split, and any depth $s+1$ of the tree. The generalization of the properties from previous section yields the following properties for a k-ary wrested fractal hand:

1. The total number of splits is $\frac{k^{s}-1}{k-1}$.

2. The total number of edges is $\frac{k^{s+1}-1}{k-1}$.

3. The total number of end-effectors is $k^{s}$.

4. The parent-pointer representation gives the array $p t=$ $\left\{0,1, \ldots, 1,2, \ldots, 2, \frac{k^{s}-1}{k-1}, \ldots, \frac{k^{s}-1}{k-1}\right\}$.

5. The total number of joints, for a wrist with $p$ joints and edges having $q$ joints each, is $j=p+\frac{k\left(k^{s}-1\right)}{k-1} q$.

Notice that the number of end-effectors grows quickly: for instance, for a ternary hand with a depth of 4,3 joints in the wrist and 2 joints per edge, the total number of joints in the hand is 81 , and the total number of end-effectors or fingertips is 27 . The study of the solvability and synthesis of general fractal hands is beyond the scope of this work.

\section{Dimensional Synthesis of Binary Hands}

The exact dimensional synthesis of a binary hand with $s$ splitting stages, or $2^{s}$ end-effectors, requires checking its solvability and, if solvable, defining the number of positions in each fingertip task in order to obtain a finite number of solutions.

\subsection{Solvability and number of solutions}

Using Equation (2), for the binary hand topology with joint array $j=\{2,1,1, \ldots, 1\}$, a few facts can be derived for this family, regardless of the number of splits:

1. The binary hand is solvable for all topologies of the family.

2. The maximum number of positions per end-effector for exact kinematic synthesis is constant and equal to three.
This can be shown as follows: the dot products of the arrays in Equation (2) are

$$
\begin{aligned}
& \mathbf{D}_{s}^{e} \cdot \mathbf{E}_{i}=8+4\left(2^{s+1}-2\right), \quad \mathbf{D}_{c}^{n} \cdot \mathbf{B}_{i}=0, \\
& \mathbf{D}_{e e}^{n} \cdot \mathbf{B}_{i}=2^{s} 6, \quad \mathbf{D}_{j}^{e} \cdot \mathbf{E}_{i}=2+\left(2^{s+1}-2\right),
\end{aligned}
$$

which yield $m=3$ regardless of the depth $s$ of the tree.

In order to conclude that the hand is solvable, we also need to check all the non-isomporphic subgraphs. Notice that the number of positions allowed for a subgraph with $b$ branches and $e$ edges is

$$
m_{i}=\frac{4}{\frac{6 b}{e+1}-1}+1
$$

which means that the most restrictive subtrees will be those with the maximum number of edges, that is, those in which we eliminate the same number of edges as branches. For a subgraph in which $i$ branches and edges have been eliminated,

$$
m_{i}=\frac{4-\frac{4 i}{2^{s+1}}}{2-\frac{5 i}{2^{s+1}}}+1
$$

where $i<2^{s}$ always and at most $i=2^{s-1}$. This number of positions tends to 3 as the number of splitting stages $s$ increases, regardless of the number of branches $i$ eliminated in the subtree. This concludes that, for all subtrees starting at the root node, the number of positions for exact synthesis is $m_{i} \geq 3$.

For those subtrees with root node substitution, the smallest of them corresponds to the $R R$ serial chain, with $m_{i}=3$, and for the rest of them we can apply the same reasoning, to conclude that the wristed binary trees are always solvable.

It is important to highlight that some of the subtrees, in particular those corresponding of two terminal edges (the edges incident on end-effectors) creating serial RR chains, have $m_{i}=3$, same as the overall number of positions. According to [9], those can be solved separately. This is the strategy followed for the synthesis of the binary hands:

1. Solve for the relative motion at the terminal edges of each two branches (a spatial RR chain) with respect to the reference frame of one of the end-effectors.

2. Once all the terminal edges are dimensioned, solve for the relative motion of the previous branching, again as spatial RR chains.

3. Continue until the last branching. Then the two wrist joints can be solved for any of the branches.

Using this methodology, the total number of solutions for a binary hand with $s$ splitting stages, or branchings, is $2^{2^{s}}$. The simplest hand, the $2-(1,1)$, has 4 solutions, and the hand topology with four splitting stages in Figure 5 yields $2^{16}$ candidate designs for $m=3$ positions. 


\subsection{Solvability for partial binary hands}

The partial binary hands obtained by pruning some of the branches are also studied for solvability. As it has been already mentioned in the previous section, the pruning at each node can either eliminate either a pair of branches or just one of them. The trees in which the pruning eliminates the pair of branches at a given node are always solvable.

The constructive proof uses the solving method presented in the previous subsection, where the hand is dimensioned by considering motion between pairs of end-effectors. A tree pruned by pairs consists of consecutive $R-R$ serial chains when considering relative motion, and hence they are solvable at each step.

Binary hands in which only one of the branches is pruned at a given node, present a heterogeneous mix of $2 R$ and $3 R$ serial chains when considering motion relative to the end-effectors. Due to this, some of the subgraphs will be more constrained in their motion than others, resulting in a tree that is not solvable for simultaneous tasks.

For those pruned binary tree that are solvable, use the same strategy and equations derived to solve for complete binary trees.

\subsection{Synthesis equations for each branching}

For each of the subproblems mentioned above, one of the several methods used to synthesize the spatial RR chain can be applied, for instance [14], [15] or [16]. It is well known that the problem yields six solutions, with only two of them being real.

The design equations for the spatial RR chain used in this work are as follows: consider the task position, expressed as a dual quaternion, $\hat{P}=\hat{p}+\varepsilon \hat{p}^{0}$, where $\hat{p}$ is the quaternion corresponding to the task rotation. Similarly, consider the joint axes as $\hat{S}_{k}=\hat{s}_{k}+\varepsilon \hat{s}_{k}^{0}=0+\mathbf{s}_{k}+\varepsilon\left(\mathbf{s}_{k}^{0}+0\right)$, a pure dual quaternion. Then the following equations can be written,

$$
\begin{aligned}
& \hat{s}_{1} \hat{p} \cdot\left(\hat{p} \hat{s}_{2}-\hat{s}_{2} \hat{p}\right)=0, \\
& \hat{s}_{1}^{0}\left(\hat{s}_{12} \hat{s}_{2}\right) \cdot \hat{p}+\hat{s}_{2}^{0}\left(\hat{s}_{1} \hat{s}_{12}\right) \cdot \hat{p}-\left(\hat{s}_{1} \hat{s}_{2}^{0}+\hat{s}_{1}^{0} \hat{s}_{2}\right) \hat{s}_{12} \cdot \hat{p} \\
& =\frac{1}{2} \hat{p}^{0} \hat{s}_{12} \cdot \hat{s}_{12}, \quad i=2,3,
\end{aligned}
$$

where $\hat{s}_{12}=\hat{s}_{1} \hat{s}_{2}-\hat{s}_{2} \hat{s}_{1}$, and the $\cdot$ is the usual dot product.

The first equation in set (7) is a scalar one, and the second is a pure quaternion equation. These eight equations can be solved for the Plucker coordinates of the axes by imposing Plucker constraints. One of the easiest solutions is obtained by finding the solution when intersecting for instance the $\mathrm{z}=1$ plane. This system of equations is easy enough to be solved using Groebner bases.

The inverse kinematics solution for the joint variables can be found similarly using

$$
\begin{aligned}
& \cos \frac{\theta_{1}}{2} \cos \frac{\theta_{2}}{2}=\frac{2\left(\hat{s}_{1} \hat{s}_{2} \hat{s}_{12}\right) \cdot \hat{p}}{\hat{s}_{12} \cdot \hat{s}_{12}} \\
& \sin \frac{\theta_{1}}{2} \cos \frac{\theta_{2}}{2}=\frac{2\left(\hat{s}_{12} \hat{s}_{2}\right) \cdot \hat{p}}{\hat{s}_{12} \cdot \hat{s}_{12}} \\
& \cos \frac{\theta_{1}}{2} \sin \frac{\theta_{2}}{2}=\frac{2\left(\hat{s}_{1} \hat{s}_{12}\right) \cdot \hat{p}}{\hat{s}_{12} \cdot \hat{s}_{12}} \\
& \sin \frac{\theta_{1}}{2} \sin \frac{\theta_{2}}{2}=\frac{-2 \hat{s}_{12} \cdot \hat{p}}{\hat{s}_{12} \cdot \hat{s}_{12}}
\end{aligned}
$$

\section{The $2-(1,1)$ Hand}

The $2-(1,1)$ hand has a two-jointed wrist and two fingers, each one consisting of a single revolute joint. Figure 8 shows the tree graph for the hand and Figure 9 shows the kinematic sketch.

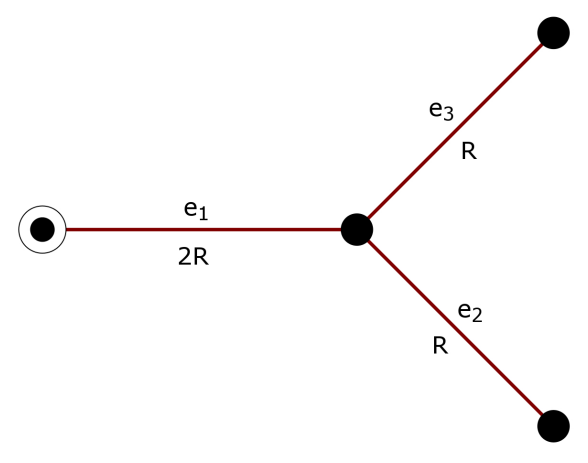

Fig. 8. Graph of the 2-(1,1) hand

Solvability calculations [10] show, as expected, that the hand is solvable for simultaneous tasks, for $m=3$ finite positions for each fingertip. In this section we show how we can reduce the rigid-body guidance problem for this hand to that of the spatial RR chain, to obtain 4 real solutions.

The design equations for each finger are created by equating the forward kinematics equations of each branch to the desired position, [16]. In what follows, $e^{\hat{S}_{j} \theta_{j}^{i}}$ denotes the screw displacement of rotation $\theta_{j}^{i}$ about joint axes $S_{j}$, regardless of the algebra used to compose the displacements.

In this case, absolute displacements from the fixed frame associated to the root node are used,

$$
\begin{aligned}
& e^{\hat{S}_{0} \theta_{0}^{i}} e^{\hat{S}_{1} \theta_{1}^{i}} e^{\hat{S}_{2} \theta_{2}^{i}} \hat{D}_{0}^{1}=\hat{P}_{i}^{1} \\
& e^{\hat{S}_{0} \theta_{0}^{i}} e^{\hat{S}_{1} \theta_{1}^{i}} e^{\hat{S}_{3} \theta_{3}^{i}} \hat{D}_{0}^{2}=\hat{P}_{i}^{2}, \quad i=1,2,3,
\end{aligned}
$$

where $\hat{D}_{0}^{j}$ represents the absolute displacement of the endeffector $j$ at a reference configuration, for each branch $j$. Notice that $\hat{P}_{i}^{j}\left(\hat{D}_{0}^{j}\right)^{-1}$ is the relative displacement of branch $j$ 


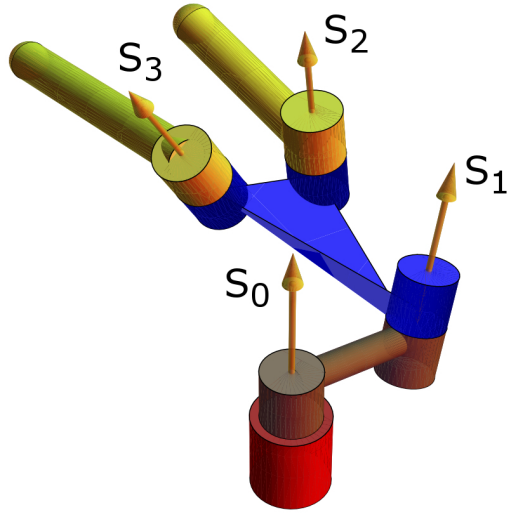

Fig. 9. Kinematic sketch of the 2-(1,1) hand

from the reference configuration up to position $\hat{P}_{i}^{j}$, expressed in the fixed frame, so that the set of equations can be written

$$
\begin{aligned}
e^{\hat{S}_{0} \theta_{0}^{i}} e^{\hat{S}_{1} \theta_{1}^{i}} e^{\hat{S}_{2} \theta_{2}^{i}}=\hat{P}_{i}^{1}\left(\hat{D}_{0}^{1}\right)^{-1} \\
e^{\hat{S}_{0} \theta_{0}^{i}} e^{\hat{S}_{1} \theta_{1}^{i}} e^{\hat{S}_{3} \theta_{3}^{i}}=\hat{P}_{i}^{2}\left(\hat{D}_{0}^{2}\right)^{-1}, \quad i=1,2,3 .
\end{aligned}
$$

In particular, if the reference configuration is taken as the first task position, the first equation is the identity and the system is reduced to reaching two relative positions.

This set of equations could be solved in order to find the dimensions of fingers and palm. However the system can be simplified to obtain an algebraic solution.

In order to simplify the set of equations to its minimum, we can write the forward kinematics of the common joints (the wrist) as a function of the rest of the chain and the task position, for each branch $j$,

$$
e^{\hat{S}_{0} \theta_{0}^{i}} e^{\hat{S}_{1} \theta_{1}^{i}}=\hat{P}_{i}^{j}\left(\hat{P}_{1}^{j}\right)^{-1} e^{\hat{S}_{j+1} \theta_{j+1}^{i}}, \quad i=2,3 ; \quad j=1,2,
$$

where $j$ indicates the branch and $i$ denotes the task position. The following system of equations is created by imposing equality of the common joints at the wrist,

$$
e^{-\hat{S}_{2} \theta_{2}^{i}} e^{\hat{S}_{3} \theta_{3}^{i}}=\left(\hat{P}_{i}^{1}\left(\hat{P}_{1}^{1}\right)^{-1}\right)^{-1} \hat{P}_{i}^{2}\left(\hat{P}_{1}^{2}\right)^{-1}, \quad i=2,3 .
$$

This equations correspond to the change of reference frame to the first end-effector in branch 1, creating a serial chain starting at the first end-effector and going to the second one. The displacement $\hat{P}_{1}^{1}\left(\hat{P}_{i}^{1}\right)^{-1} \hat{P}_{i}^{2}\left(\hat{P}_{1}^{2}\right)^{-1}$ corresponds to the relative displacements from the first position to position $i$, measured in the first end-effector frame.
The set in Eq.(12) corresponds to the equations obtained for a spatial RR chain. Solve for the axes using Eq.(7) to obtain two solution for axes $S_{2}$ and $S_{3}$. Once those two solutions are found, the wrist axes can be solved as another spatial RR chain in the previous sets of equations, Eq.(11). Each solution of the $S_{2}$ and $S_{3}$ axes will yield two solutions for the $S_{0}$ and $S_{1}$ axes, for a total of four hand designs.

\section{The 2-(1-(1,1),1-(1,1)) Hand}

This hand topology has two splitting stages and four fingertips. Figure 10 shows the tree graph and Fig 11 shows the kinematic sketch.

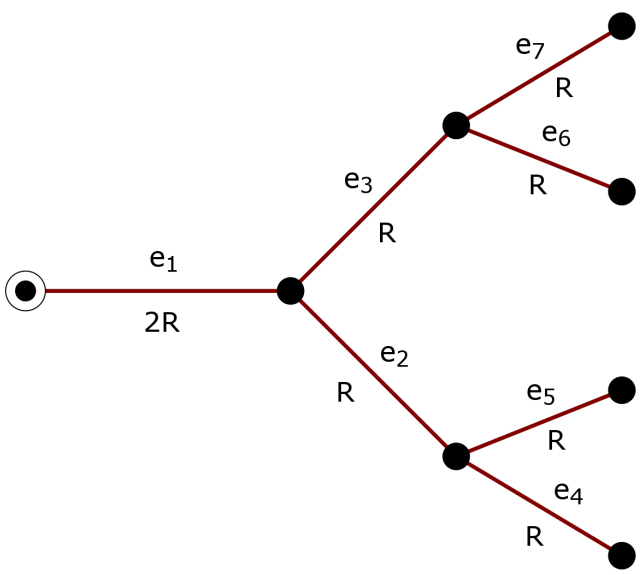

Fig. 10. Graph of the 2-(1-(1,1),1-(1,1)) hand

The synthesis for $m=3$ finite positions per fingertip follows the steps described in Section 5.

The relative positions to reach,

$$
\begin{aligned}
& e^{\hat{S}_{0} \theta_{0}^{i}} e^{\hat{S}_{1} \theta_{1}^{i}} e^{\hat{S}_{2} \theta_{2}^{i}} e^{\hat{S}_{4} \theta_{4}^{i}}=\hat{P}_{1 i}^{1}, \\
& e^{\hat{S}_{0} \theta_{0}^{i}} e^{\hat{S}_{1} \theta_{1}^{i}} e^{\hat{S}_{2} \theta_{2}^{i}} e^{\hat{S}_{5} \theta_{5}^{i}}=\hat{P}_{1 i}^{2}, \\
& e^{\hat{S}_{0} \theta_{0}^{i}} e^{\hat{S}_{1} \theta_{1}^{i}} e^{\hat{S}_{3} \theta_{3}^{i}} e^{\hat{S}_{6} \theta_{6}^{i}}=\hat{P}_{1 i}^{3}, \\
& e^{\hat{S}_{0} \theta_{0}^{i}} e^{\hat{S}_{1} \theta_{1}^{i}} e^{\hat{S}_{3} \theta_{3}^{i}} e^{\hat{S}_{7} \theta_{7}^{i}}=\hat{P}_{1 i}^{4}, \quad i=2,3,
\end{aligned}
$$

can be transformed into two sets of equations in order to dimension the terminal edges for the relative motion between them,

$$
\begin{aligned}
& e^{-\hat{S}_{4} \theta_{4}^{i}} e^{\hat{S}_{5} \theta_{5}^{i}}=\left(\hat{P}_{1 i}^{1}\right)^{-1} \hat{P}_{1 i}^{2}, \\
& e^{-\hat{S}_{6} \theta_{6}^{i}} e^{\hat{S}_{7} \theta_{7}^{i}}=\left(\hat{P}_{1 i}^{3}\right)^{-1} \hat{P}_{1 i}^{4}, \quad i=2,3 .
\end{aligned}
$$

Each of these sets of equations are solved independently to obtain the joint axes $S_{4}, S_{5}, S_{6}$ and $S_{7}$ and the corresponding rotation angles, using the spatial RR solution method. 


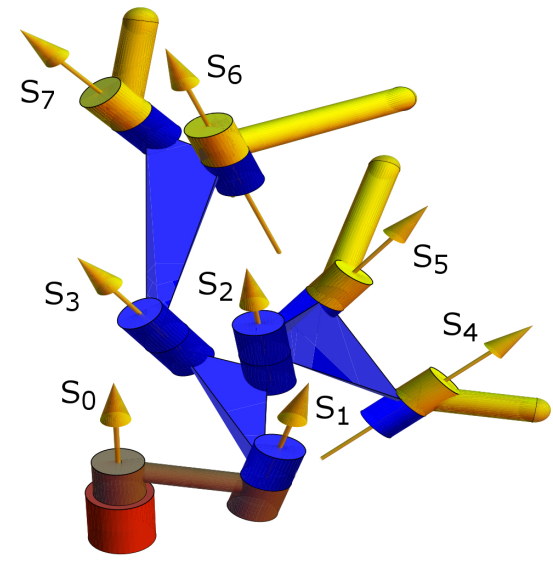

Fig. 11. Kinematic sketch of the 2-(1-(1,1),1-(1,1)) hand

Use the solutions to dimension the joint axes of the first branching stage, selecting two of the four end-effector poses,

$$
e^{-\hat{S}_{2} \theta_{2}^{i}} e^{\hat{S}_{3} \theta_{3}^{i}}=\left(\hat{P}_{1 i}^{1} e^{-\hat{S}_{4} \theta_{4}^{i}}\right)^{-1} \hat{P}_{1 i}^{6} e^{-\hat{S}_{6} \theta_{6}^{i}}, \quad i=2,3 .
$$

After this, any of the four end-effector chains can be used to dimension the remaining two axes, corresponding to the wrist, for instance

$$
e^{\hat{S}_{0} \theta_{0}^{i}} e^{\hat{S}_{1} \theta_{1}^{i}}=\hat{P}_{1 i}^{1} e^{-\hat{S}_{4} \theta_{4}^{i}} e^{-\hat{S}_{2} \theta_{2}^{i}}, \quad i=2,3 .
$$

The output of the process is a set of 16 candidate solutions.

\section{The Binary Hand with $n$ End-effectors}

The synthesis presented in Sections 6 and 7 can be generalized for any number of splitting stages $s$. The relative positions for the joints incident on end-effectors are, for a binary hand $\left\{0,1,1,2,2,3,3, \ldots 2^{s}-1,2^{s}-1\right\}$ with $s$ splitting stages and $2^{s}$ fingertips,

$$
\begin{aligned}
e^{-\hat{S}_{k} \theta_{k}^{i}} e^{\hat{S}_{k+1} \theta_{k+1}^{i}}=\left(\hat{P}_{1 i}^{f}\right)^{-1} \hat{P}_{1 i}^{f+1}, \quad & i=2,3, \\
k & =2^{s}, 2^{s}+2, \ldots, 2^{s+1}-2, \\
& f=1,3, \ldots, b-1
\end{aligned}
$$

with $k$ enumerating the end-effector joints, from $2^{s}$ to $2^{s+1}-1$, corresponding to end effectors indicated by superscript $f$.

These equations are solved for the corresponding Plucker coordinates of the joint axes and for the joint angles, using Eqs.(7) and (8).
After that, the previous splitting stage is solved with

$$
\begin{aligned}
e^{-\hat{S}_{k} \theta_{k}^{i}} e^{\hat{S}_{k+1} \theta_{k+1}^{i}}=\left(\hat{P}_{1 i}^{f} e^{-\hat{S}_{m} \theta_{m}^{i}}\right)^{-1} \hat{P}_{1 i}^{g} e^{-\hat{S}_{r} \theta_{r}^{i}} \\
\quad i=2,3, \\
k=2^{s-1}, 2^{s-1}+2, \ldots, 2^{s}-2,
\end{aligned}
$$

where $\mathrm{k}$ enumerates the joints corresponding to this splitting stage, and we have some flexibility in the selection of the end-effectors $f$ and $g$ and associated joint axes $m$ and $r$ to be used to create the kinematic chain containing axes $k$ and $k+1$. This process is repeated up to the wrist joints, which can be solved using any of the serial chains leading to an end-effector, for instance the first one,

$$
e^{\hat{S}_{0} \theta_{0}^{i}} e^{\hat{S}_{1} \theta_{1}^{i}}=\hat{P}_{1 i}^{1} e^{-\hat{S}_{2} \theta_{2 s}^{i}} \ldots e^{-\hat{S}_{2} \theta_{2}^{i}}, \quad i=2,3 .
$$

\subsection{Candidate solution sorting and ranking}

An important post-synthesis step in the design process is how to rank the $2^{2^{s}}$ candidate solutions for a binary hand of depth $s+1$ in order to select the most suited solution for the task.

In finding an optimal solution, it is important to distinguish between requirements that depend on the dimensions of the joint axes, and requirements that are defined by the placement and shape of the links. There is some freedom in the link dimensioning and placement for spatial linkages given the position of the joints, so that links can be optimized separately in a post-synthesis, link-based optimization process [17]. Some of the requirements that can be targeted using the link optimization are link and joint lengths and force transmission at the joint level. Some other performance requirements are dependent on the position of the joints, such as singularities, and some others depend both on the position of the joints and the links, such as overall dimensions, self-intersections and obstacle avoidance.

For an optimal solution, a possible strategy is to perform approximate synthesis with additional performance requirements. Those requirements will in general reduce the number of suitable candidates, so an important first step is the knowledge of the number of potential designs available to the designer, which is one of the results of this work. In the case of multi-fingered robotic hand, interesting performance requirements may include graspability and manipulability conditions, according to the task being targeted.

\section{Design Example}

The synthesis results presented here for binary hands allow following several design strategies. One possible strategy arises from noticing that the difference between the motion of two consecutive end-effectors happens at the terminal edges. The binary hand can be designed by pre-designing the relative motion between those fingertips and using the previous joints to locate that action. In this section we present one example that locates two gripping end-effectors for a given task. 
For this example, the $1-(1-(1,1), 1-(1,1))$ topology is used, where the last branching stages are pre-designed and kinematic synthesis is applied for designing the first branching stage as well as the wrist joints.

The two pairs of fingertips, $1-2$ and $3-4$, are designed with parallel joint axes. Figure 12 shows the selected design and the computation of the motion between both endeffectors expressed in the frame attached to one of them, for the case of branches 1 and 2 .

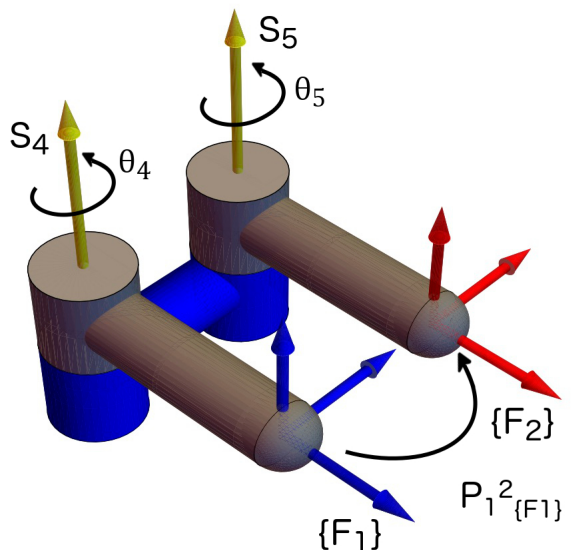

Fig. 12. Fingertip design and motion with respect to Frame 1

Table 1 shows the selected relative motion between fingertips 1 and 2, and 3 and 4, expressed as the motion of fingertip 2 seen from fingertip 1, and the motion of fingertip 4 seen from fingertip 3 . Table 2 shows the three positions for each of the four fingertips, as expressed in the fixed frame. Notice that, in this case, the fixed frame is attached to the arm holding the 2-dof wrist.

Table 1. Relative positions between fingertips

\begin{tabular}{c} 
Fingers 1 and 2 \\
\hline $0.71 i+0.71 j+\varepsilon(-1.41 i+1.41 j)$ \\
$0.1+0.7 i+0.7 j-0.1 k+\varepsilon(-0.89 i+0.89 j-0.071 k-0.071)$ \\
$0.1+0.7 i+0.7 j-0.1 k+\varepsilon(-0.89 i+0.89 j+0.071 k+0.071)$ \\
\hline Fingers 3 and 4 \\
\hline $0.71 i+0.71 j+\varepsilon(-1.41 i+1.41 j)$ \\
$-0.14+0.69 i+0.69 j+0.14 k+\varepsilon(-2.1 i+2.1 j)$ \\
\hline $0.66 i+0.66 j+0.24 k+\varepsilon(-2.6 i+2.6 j-0.071 k-0.071)$
\end{tabular}

Table 2. Task positions for each fingertip

\begin{tabular}{c} 
Finger 1 \\
\hline $0.71-0.71 j+\varepsilon(7.07 j+7.07)$ \\
$0.66-0.24 i-0.66 j+0.24 k+\varepsilon(-0.07 i+7.6 j+4.9 k+5.7)$ \\
$0.39-0.58 i-0.68 j-0.19 k+\varepsilon(-1.3 i+2.2 j+12.0 k+7.7)$ \\
\hline Finger 2 \\
\hline $0.5+0.5 i+0.5 j+0.5 k+\varepsilon(4.0 i+6.0 j-6.0 k-4.0)$ \\
$0.73+0.34 i+0.54 j+0.25 k+\varepsilon(-0.97 i+8.6 j ? 6.3 k-3.7)$ \\
$0.91+0.42 i+0.009 j+0.004 k+\varepsilon(-3.6 i+14.0 j ? 3.1 k+1.5)$ \\
\hline Finger 3 \\
\hline $1+\varepsilon(-8.84 i+8.84 j)$ \\
$0.99-0.13 i+\varepsilon(-12.0 i+8.8 j+1.1 k-1.7)$ \\
\hline $0.88-0.12 i+0.061 j+0.45 k+\varepsilon(-7.2 i+14.0 j+3.3 k-3.6)$ \\
\hline $0.71 i+0.71 j+\varepsilon(-1.41 i+1.41 j-12.50 k)$ \\
$-0.28+0.33 i+0.9 j-0.014 k+\varepsilon(-2.9 i-0.72 j-16.0 k-4.9)$ \\
\hline
\end{tabular}

The design process yields four candidate designs, and one of them is selected as the final design. This selection is based on a quick modeling and simulation of the four candidate solutions. Table 3 shows the Plucker coordinates of the axes and Figure 13 shows the CAD model of the hand at the three different positions.

Table 3. Plucker coordinates of the joint axes, according to the joint notation in Figure 11

\begin{tabular}{|c|c|}
\hline Axis & Plucker coordinates \\
\hline $\mathrm{S}_{0}$ & $(0.44,-0.54,0.71)+\varepsilon(13.0,12.0,1.2)$ \\
$\mathrm{S}_{1}$ & $(0.08,-0.80,0.59)+\varepsilon(5.5,7.8,9.9)$ \\
$\mathrm{S}_{2}$ & $(-0.2,0.005,-0.98)+\varepsilon(-13.0,-4.3,2.7)$ \\
$\mathrm{S}_{3}$ & $(-0.99,0.03,0.14)+\varepsilon(2.6,2.5,18.0)$ \\
$\mathrm{S}_{4}$ & $(0.0,0.0,1.0)+\varepsilon(15.0,0.0,0.0)$ \\
$\mathrm{S}_{5}$ & $(0.0,0.0,1.0)+\varepsilon(15.0,4.0,0.0)$ \\
$\mathrm{S}_{6}$ & $(1.0,0.0,0.0)+\varepsilon(0.0,0.0,-12.68)$ \\
$\mathrm{S}_{7}$ & $(1.0,0.0,0.0)+\varepsilon(0.0,4.0,-12.68)$ \\
\hline
\end{tabular}

\section{Conclusions}

Fractal hands present several splitting stages, each of them splitting in the same number of branches. Here we present the design of wristed binary hands, those multi- 

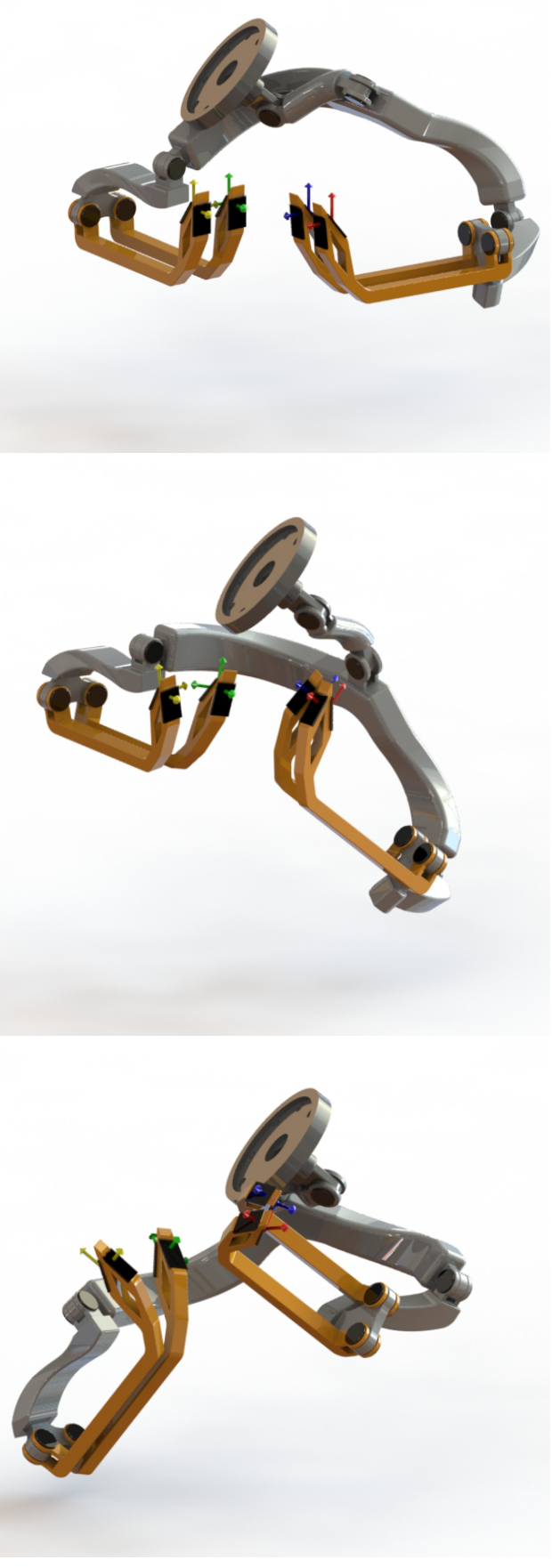

Fig. 13. Final hand design with fingertips reaching task positions 1, 2 and 3 . The task positions are indicated with reference frames.

fingered hands that split in two branches at each stage. Some properties of the binary hands are derived and the solvability of all possible topologies in this family is calculated. Design equations are created for the general case, based on iteratively dimensioning the relative motion of branches, starting at the end-effectors. It is also derived that other less symmetric hand topologies can be created by pruning the binary hands. The dimensional synthesis equations are derived in particular for hands with one and two splitting stages, and also for the general case. One design example illustrates the synthesis process.
Binary hands are one of the many families of hand topologies that can be enumerated and solved for a specific task or application. Even though it has been proved that there is an infinite number of possible designs that can be synthesized, some families of hands can be fully characterized, as in the case of wristed binary hands.

These binary hands may have applications in multiple, simultaneous mini-manipulation and in manipulation of objects at different scales, for which the stages can be added in synchronous motion to create more sizable fingers. The use of a systematic methodology for the design of multi-fingered hands may lead to optimized end-effectors for specific sets of tasks.

\section{Acknowledgements}

This work is supported by the National Science Foundation under Grant No. 1208385. The content is solely the author's responsibility.

\section{References}

[1] Stramigioli, S., 2001, Modeling and IPC control of interactive mechanical systems - A coordinate-free approach, vol. LNCIS 266, Springer.

[2] Tischler, C., Samuel, A., and Hunt, K., 1995, "Kinematic chains for robot hands - 1. orderly number synthesis," Mechanism and Machine Theory, 30(8), pp. 1193-1215.

[3] Chen, I., Yang, G., and Kang, I., 1999, "Numerical inverse kinematics for modular reconfigurable robtos," Journal of Robotic Systems, 16(4), pp. 213-225.

[4] Garcia de Jalon, J. and Bayo, E., 1994, Kinematic and Dynamic Simulation of Multibody Systems: The RealTime challenge, Springer-Verlag.

[5] Jain, A., 2010, "Graph-theory roots of spatial operators for kinematics and dynamics," Proc. of the 2010 International Conference on Robotics and Automation, Anchorage, Alaska, USA, pp. 2745-2750.

[6] Lee, J.-J. and Tsai, L., 2002, "Structural synthesis of multi-fingered hands," ASME Journal of Mechanical Design, pp. 272-276.

[7] Simo-Serra, E., Moreno-Noguer, F., and PerezGracia, A., August 29-31, 2011, "Design of Nonanthropomorphic Robotic Hands for Anthropomorphic Tasks," ASME Design Engineering Technical Conferences, Washington DC, USA.

[8] Simo-Serra, E., Perez-Gracia, A., Moon, H., and Robson, N., June 2012, "Design of multi fingered robotic hands for finite and infinitesimal tasks using kinematic synthesis," Advances in Robot Kinematics, Innsbruck, Austria.

[9] Simo-Serra, E. and Perez-Gracia, A., 2014, "Kinematic synthesis using tree topologies," Mechanism and Machine Theory, 72 C, pp. 94-113.

[10] Makhal, A. and Perez-Gracia, A., June 2014, "Solvable multi-fingered hands for exact kinematic synthesis ," Advances in Robot Kinematics, Ljubljiana, Slovenia. 
[11] Selig, J. M., 2004, Geometric Fundamentals of Robotics (Monographs in Computer Science), SpringerVerlag.

[12] Tsai, L. W., 2001, Mechanism Design: Enumeration of Kinematic Structures According to Function, CRC Press, Boca Raton.

[13] Ma, R. R. and Dollar, A. M., 2011, "On dexterity and dexterous manipulation," The 15th International Conference on Advanced Robotics.

[14] Mavroidis, C., Lee, E., and Alam, M., 2001, "A new polynomial solution to the geometric design problem of spatial rr robot manipulators using the denavithartenberg parameters," ASME Journal of Mechanical Design, 123, pp. 58-67.

[15] Perez-Gracia, A. and McCarthy, J. M., 2003, "Dimensional synthesis of bennett linkages," ASME Journal of Mechanical Design, 125, pp. 98-104.

[16] Perez-Gracia, A. and McCarthy, J. M., 2006, "Kinematic synthesis of spatial serial chains using clifford algebra exponentials," Proceedings of the Institution of Mechanical Engineers, Part C: Journal of Mechanical Engineering Science, 220(7), pp. 953-968.

[17] Yihun, Y., Bosworth, K., and Perez-Gracia, A., 2014, "Link-based performance optimization of spatial mechanisms," Journal of Mechanical Design, 136(12). 